\section{Calibration of the Fabry- Pérot free spectral range using a tunable laser in a Michelson interferometer}

\author{
Alexandre Pereira Cabral, MEMBER SPIE, and \\ José Manuel Rebordão, MEMBER SPIE \\ National Institute of Engineering, Technology and \\ Innovation (INETI), 1649-038 Lisbon, Portugal
}

\begin{abstract}
Coherent interferometric absolute distance metrology enables different techniques for length metrology. Measurements are made without ambiguity, by using either one or several synthetic wavelengths resulting from the beating of two or more wavelengths or, in the case of frequency sweeping interferometry $(\mathrm{FSI})$, from a frequency sweep. These techniques require accurate measurements of the frequency variation, a task which is usually performed with a Fabry-Pérot (FP) interferometer. Sensor performances are highly dependent on the FP free spectral range (FSR), which must be accurately known. We describe a method to measure the FSR of an FP using a tunable laser in a Michelson interferometer, using both absolute and relative distance measurements. The optical setup is based on a frequency-sweeping sensor and a controlled translation table. An uncertainty of $45 \mathrm{ppm}$ in the measurement of a $1-\mathrm{GHz}$ FSR is obtained. The method is indicated for the calibration of the FP in the frequency-sweep-range-measuring subsystem of a frequency sweeping sensor absolute distance sensor as it does not require any addition hardware, configuring a self-calibration approach. () 2006 Society of PhotoOptical Instrumentation Engineers.

[DOI: 10.1117/1.2359427]
\end{abstract}

Subject terms: Fabry-Pérot; metrological instrumentation; interferometry; tunable lasers.

Paper 060357L received May 11, 2006; accepted for publication Jul. 27, 2006; published online Oct. 11, 2006.

\section{Introduction}

Absolute distance metrology is required for a wide gamut of applications, each with different ranges and resolutions requirements. Clear examples are the recent space missions comprising independent satellites working cooperatively; the management of such formations requires suitable metrology to keep the formation coherent and to enable guidance and navigation for the complete formation. ${ }^{1}$

Some techniques to measure absolute distances by interferometry exist that do not require any moving optical components. Frequency sweeping interferometry (FSI) is one of them, generating a synthetic wavelength (much longer than the optical wavelength) that increases with decreasing sweep range. ${ }^{2-4}$ The technique is not new, dating back (at least) to the 1980's (Ref. 5), but was not studied extensively until the development of tunable lasers and the emergence of external cavity diode lasers (ECDLs).

FSI does not require stabilized laser sources and relies

0091-3286/2006/\$22.00 @ 2006 SPIE only on a tunable laser and a frequency sweep range measurement subsystem, normally based on a Fabry-Pérot interferometer $(\mathrm{FP})$. The synthetic wavelength $(\Lambda)$ is inversely proportional to the frequency sweep range $(\Delta \nu)$ :

$\Lambda=\frac{c}{\Delta \nu}$,

where $c$ is the speed of light. The sweep range measurement is obtained by multiplying the FP free spectral range (FSR) by the number of resonances detected while the laser frequency sweeps. The beginning of the sweep is determined by a particular cavity mode; the laser sweeps from mode to mode and the number of resonances, separated by the FP FSR, is detected and counted. The frequency sweep range is then given by

$\Delta \nu=r \mathrm{FSR}$,

where $r$ is the number of detected FSRs detected (number of resonances - 1). The synthetic wavelength is inversely proportional to the FSR:

$\Lambda=\frac{c}{r \mathrm{FSR}}$.

The uncertainty of the distance to be measured is highly influenced by the uncertainty in $\Lambda$, especially if the measured distance corresponds to a huge number of $\Lambda$. The uncertainty in $\Lambda$ has two components: the uncertainty in $r$, corresponding to the accuracy on the determination of resonance position; and the uncertainty in the FSR value. The first one is directly determined by system performances. The second one must be determined with an accuracy of the same order of the required uncertainty of the distance measurement. Thus, in addition to the fact that the stability of the FP FSR must be ensured, it is mandatory to know its exact value. As an example, for an absolute sensor tuned to measure up to $100 \mathrm{~mm}$ with an uncertainty below $5 \mu \mathrm{m}$, corresponding to $50 \mathrm{ppm}$, the FP FSR of $1 \mathrm{GHz}$ must be known with an uncertainty of at least $50 \mathrm{kHz}$.

Different techniques exist to calibrate the FP FSR, and some provide uncertainties ${ }^{6}$ in the order of $0.1 \mathrm{ppm}$, but they normally require dedicated and sometimes complex additional hardware. We propose a simple method to calibrate the FP FSR using only the absolute metrology FSI setup, a kind of FSI FP self-calibration.

\section{FP FSR Calibration by FSI}

FSI measures the absolute value of the optical path difference (OPD) between the two arms of a Michelson interferometer (Fig. 1).

While the frequency sweeps, the detection electronics count the number of fringes (both the integer part and phase), from the beginning to the end of the sweep without ambiguity. In parallel, the frequency sweep is measured by counting FP resonances. The measured length is given by

$L_{\mathrm{ABS}}=\frac{N_{\mathrm{ABS}}}{2} \frac{\Lambda}{n}=\frac{N_{\mathrm{ABS}}}{2} \frac{c}{r \mathrm{FSR} n}$,

where $N_{\mathrm{ABS}}$ is the number of synthetic wavelength fringes, and $n$ the refractive index of the air. It is also possible to 


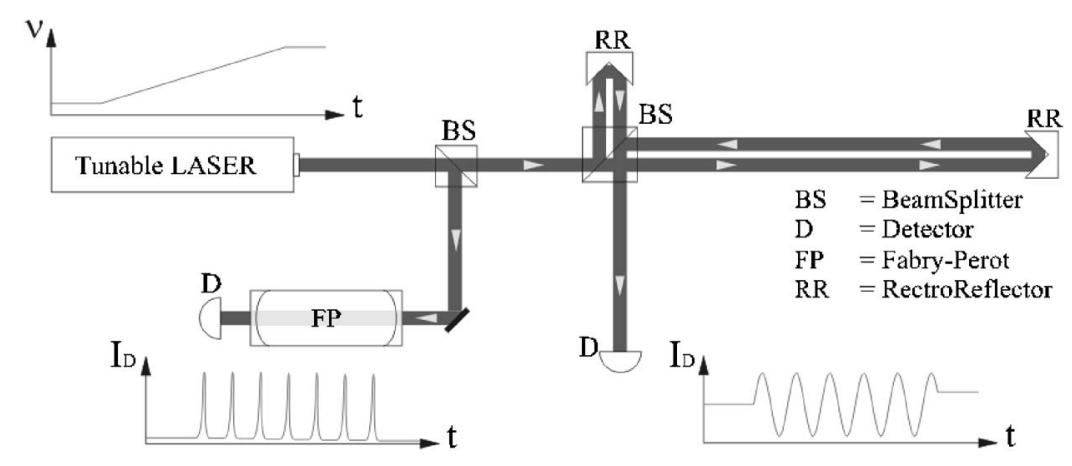

Fig. 1 FSI setup based on a Michelson interferometer and an FP to measure the frequency sweep range.

measure a (relative) displacement using the same interferometric setup and a laser operated in a single known frequency. In this case, the length variation is given by

$\Delta L_{\mathrm{REL}}=\frac{N_{\mathrm{REL}}}{2} \frac{\lambda}{n}$,

where $N_{\text {REL }}$ is the number of optical fringes and $\lambda$ the optical wavelength. If we perform two absolute measurements at different positions ( 1 and 2), the separation is given by

$\Delta L_{12}=\frac{c}{2 \mathrm{FSR} n}\left(\frac{N_{\mathrm{ABS} 2}}{r_{2}}-\frac{N_{\mathrm{ABS} 1}}{r_{1}}\right)$.

Consequently, if we count the number of relative optical fringes $\left(N_{\mathrm{REL}}\right)$ between positions 1 and 2, the FP FSR can be determined from Eqs. (5) and (6):

$\mathrm{FSR}=\frac{c}{\lambda N_{\mathrm{REL}}}\left(\frac{N_{\mathrm{ABS} 2}}{r_{2}}-\frac{N_{\mathrm{ABS} 1}}{r_{1}}\right)$

The method was used to calibrate a temperature stabilized confocal FP with a FSR of $1 \mathrm{GHz}$ (FP100 from TOPTICA). The optical setup implemented is sketched in Fig. 2. It consists of an absolute distance sensor based on FSI and a controllable translation table carrying one of the interferometer retroreflectors (RRs). The tunable laser is an

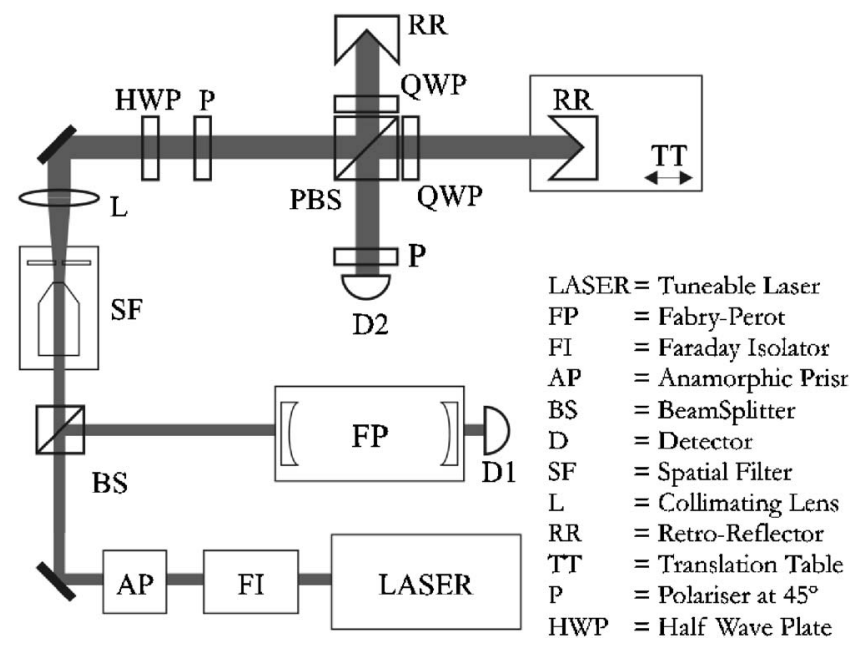

Fig. 2 Optical setup of the implemented FSI sensor.
ECDL capable of a mode-hop-free sweep range up to $150 \mathrm{GHz}$ (TBL-7000 from NewFocus in a Littman-Metcalf mounting at $633 \mathrm{~nm}$ ), corresponding to a synthetic wavelength around $2 \mathrm{~mm}$. A Faraday isolator (FI) prevents feedback from the interferometer components back to the laser and an anamorphic prism pair (AP) circularizes the elliptical cross section of the beam. The beam is then split by the first beamsplitter (BS): one part is reflected to the FP and the remainder is transmitted to the interferometer (in a polarizing configuration).

A homodyne detection scheme is used. During the measurement, while the frequency sweeps, the signals from the detectors (FP and interferometer) are acquired simultaneously. As the maximum number of fringes for the required range is small, it is conceivable to acquire all the data for postprocessing. After signal processing, we obtain the number of fringes per resonance $N_{\mathrm{ABS}} / r$.

\section{Experiments and Results}

The evaluation of the FSR calibration measurement uncertainty was made according to the Guide to the Expression of Uncertainty in Measurement. ${ }^{7}$ Figure 3 shows the results obtained for an expanded uncertainty of the measurement corresponding to a coverage probability of approximately $95 \%$, for measurements made at separations from 25 to $150 \mathrm{~mm}$. The contribution to the standard uncertainty associated with $N_{\text {REL }}$ and $\lambda=632.908 \mathrm{~nm}$ are, respectively, 1 fringe and $0.003 \mathrm{~nm}$. The contribution associated with the measurement of $N_{\mathrm{ABS}} / r$, corresponding to more than $90 \%$ of the final uncertainty, was determined statistically from 100 measurements at each separation (the uncertainty of this absolute measurement is around $5 \mu \mathrm{m}$ ).

For a separation of $150 \mathrm{~mm}$, the value of the FP FSR was $1001.178 \pm 0.045 \mathrm{MHz}$, corresponding to a relative uncertainty of $45 \mathrm{ppm}$. The solid curve in the top graph of Fig. 3 represents the predicted uncertainty using the mentioned experimental parameters for separations between $10 \mathrm{~mm}$ and $1 \mathrm{~m}$. Thus, it seems possible to achieve an uncertainty below $10 \mathrm{ppm}$ for a separation of $1 \mathrm{~m}$.

Summarizing, we showed that it is possible to calibrate the FP FSR with an uncertainty of the order of tens of parts per million. The optical setup comprises an absolute distance sensor based on FSI and a controllable translation table. In the case of FSI absolute sensors, this technique is highly indicated as it does not require additional complex hardware. For demanding space applications, requiring 


\section{OE LETTERS}
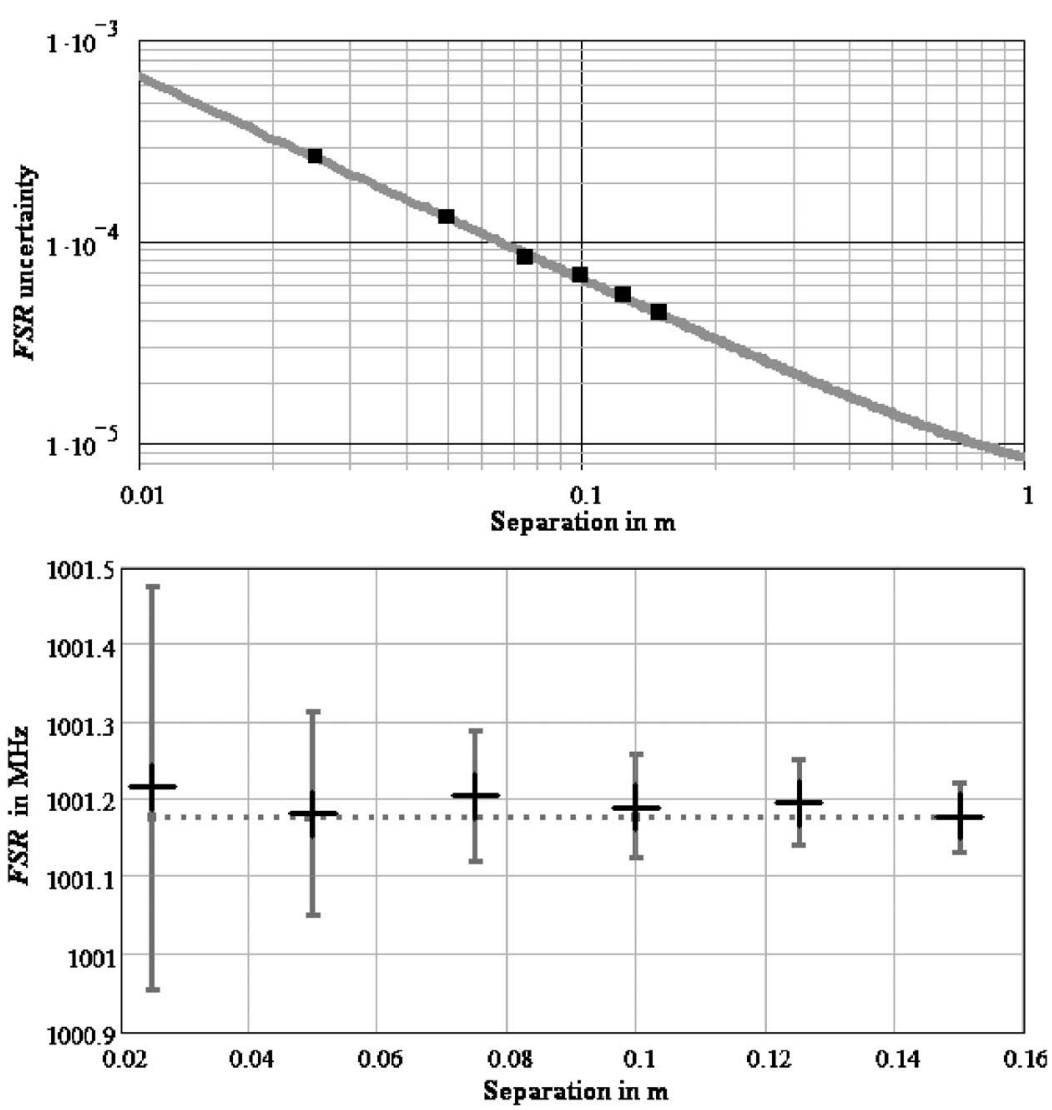

Fig. 3 FP calibration results.

calibrations after launch, when the satellite reaches its final location, this technique can be easily implemented, avoiding additional complexity.

The results obtained were tuned for our FSI absolute sensor with 5 - $\mu \mathrm{m}$ uncertainty. Lower uncertainties can be obtained with more accurate absolute sensors and larger separations.

\section{References}

1. B. Calvel, I. Cabeza, A. Cabral, J. Rebordão, E. Manske, R. Sesselmann, Z. Sodnik, and A. Verlaan, "High precision optical metrology for DARWIN: design and performances," in Proc. 5th Int. Conf. on Space Optics, pp. 501-507, ESA (2004).

2. D. Xiaoli and S. Katuo, "High-accuracy absolute distance measure- ment by means of wavelength scanning heterodyne interferometry," Meas. Sci. Technol., 9, 1031-1035 (1998).

3. B. Swinkels, N. Bhattacharya, A. Wielders, and J. Braat, "Absolute distance metrology for space interferometers," in Optical Measurement Systems for Industrial Inspection IV, W. Osten, C. Gorecki, and E. Novak, Eds., Proc. SPIE 5856, 312-317 (2005).

4. A. Cabral and J. Rebordão, "Absolute distance metrology with frequency sweeping interferometry," in Recent Developments in Traceable Dimensional Measurements III, J. Decker and G.-S. Peng, Eds., Proc. SPIE 5879, 195-204 (2005).

5. H. Kikuta, K. Iwata, and R. Nagata, "Distance measurement by the wavelength shift of laser diode light," Appl. Opt. 25, 2976-2980 (1986).

6. P. Manson, "High precision free spectral range measurement using a phase modulated laser beam," Rev. Sci. Instrum. 70, 3834-3839 (1999).

7. IOS, Guide to the Expression of Uncertainty in Measurement International Organization for Standardization, Geneva (1995). 